\title{
DEMENTIA AND MILD COGNITIVE IMPAIRMENT IN PARKINSON'S DISEASE
}

Arq Neuropsiquiatr 2009;67(3-B):957-959

\section{THE AUTHORS' REPLY}

We are grateful to Caixeta et al. ${ }^{1}$ for their interest in our article and for the comments and questions they raised.

The fundamental question was that:

"The use of CERAD (Consortium to Establish a Registry for Alzheimer' Disease) is indicated to Alzheimer' Disease... being unsuitable for subcortical dementia such as Parkinson's disease dementia" "CERAD battery is sensitive to capture limbic amnesia, apraxia, agnosia and naming deficits, which are relatively common in Alzheimer's disease but rarer in subcortical forms of dementia, and.... less sensitive to detect some executive dysfunction which are very common in Parkinson's disease dementia"

We would like to raise the following considerations with respect to this question:

1) It is not really adequate to label Parkinson's disease (PD) dementia as "subcortical", since when there is dementia in PD, the post-mortem examinations show an association with limbic or neocortical Lewy bodies pathology and neuroimaging studies have revealed considerable cortical grey matter reductions ${ }^{2}$.

2) Amnesia is not rare in Parkinson's disease and can occur in a significant proportion of the patients, even at the start of the disease. CERAD includes evaluations of word list repetition, recall and recognition, making it possible to evaluate differences in performance between recall and recognition. Our finding of difficulties in both recall and recognition suggest the existence of mechanisms analogous to those of Alzheimer's disease, in agreement with recent studies ${ }^{3}$.

3) Verbal fluency is part of CERAD, and is the most studied of the executive cognitive tests in $\mathrm{PD}^{3}$, showing elevated correlation with the more specific tests, and is thus indicated as a valuable tool for assessing executive functions in patients with PD 4 .

4) In essence, CERAD includes the elements of "Diagnostic procedures for Parkinson's disease dementia: recommendations from the Movement Disorder Society Task Force" which are, respectively: for attention, the serial 7s of the Mini-mental State Examination (MMSE); executive function, verbal fluency; visuoconstructive ability, MMSE pentagons; and memory, word recall of the MMSE ${ }^{5}$.

5) Other cognitive aspects could have been studied in our work, but the characterization of mild cognitive impairment could have been made difficult by the lack of national valida- tion of the instruments ${ }^{4}$ or of more profound studies on clinical use, sensibility and specificity ${ }^{3}$.

With respect to the possibility that the anti-parkinsonian agents could have caused mild cognitive impairment or dementia, our patients were taking levodopa, and there is no evidence that this drug can cause significant cognitive deficits ${ }^{6}$.

On the other hand there is no foundation for the supposition that, in our casuistic, the motor features of PD, which frequently affect the daily activities, could have caused an impact on the Pfeffer questionnaire scores increasing the diagnosis of dementia, since this questionnaire was not a fundamental element in this diagnosis.

\section{REFERENCES}

1. Caixeta L, Soares VL, Soares CD, Reis G. Dementia and mild cognitive impairment in Parkinson's disease. Opinion. Arq Neuropsiquiatr 2009;67:959.

2. Farlow MR, Cummings J. A modern hypothesis: the distinct pathologies of dementia associated with Parkinson's disease versus Alzheimer' disease. Dement Geriatr Cogn Disord 2008;25:301-308.

3. Emre M, Aarsland D, Brown R, et al. Clinical diagnostic criteria for dementia associated with Parkinson's disease. Mod Disord 2007;12:1689-1707.

4. Sobreira EST, Pena MCS, Silva Filho JH, et al. Executive cognitive tests for the evaluation of patients with Parkinson's disease. Dementia \& Neuropsychologia 2008;2:206-210.

5. Dubois B, Burn D, Goetz C, et al. Diagnostic procedures for Parkinson's disease dementia: recommendations from the Movement Disorder Society Task Force. Mov Disord 2007;22: 2314-2324.

6. Molloy SA, Rowan EN, O'Brien JT, McKeith IG, Wesnes K, Burn DJ. Effect of levodopa on cognitive function in Parkinson's disease with and without dementia and dementia with Lewy bodies. J Neurol Neurosurg Psychiatry; 2006;77(12):1323-1328.

\author{
Gloria Maria Almeida Souza Tedrus \\ Lineu Corrêa Fonseca \\ Grace Helena Letro \\ Alexandre Souza Bossoni \\ Adriana Bastos Samara
}




\section{DEMENTIA AND MILD COGNITIVE IMPAIRMENT IN PATIENTS WITH PARKINSON'S DISEASE}

Tedrus et al. recently published in Arq Neuropsiquiatr $(2009 ; 67: 423-427)$ an interesting study evaluating the occurrence of cognitive impairment in 32 subjects with Parkinson's disease $(\mathrm{PD})^{1}$. The authors concluded that cognitive dysfunction occurred in a significant percentage of PD patients: dementia in $21.8 \%$ and mild cognitive impairment in $31.2 \%$. Another important conclusion of this study was the relationship between schooling level and motor disability with cognitive dysfunction'. In fact cognitive dysfunction is common in PD and comprehends attentional, executive, visuospatial and memory deficits ${ }^{2}$. Caviness et al. studied mild cognitive impairment (MCl) in PD and they found that the cognitive domain most frequently abnormal in PD-MCI was frontal/executive dysfunction followed by amnestic deficit ${ }^{3}$. Dementia in PD is characterized predominantly by a progressive dysexecutive syndrome, as a result of frontal dysfunction secondary to cortical and subcortical changes due to dopaminergic and cholinergic deficiency ${ }^{4}$. In summary, Tedrus et al. concluded that cognitive compromise in PD is of great clinical importance and we think that their results could be compared with others studies previously performed in Brazil ${ }^{5}$.

\section{REFERENCES}

1. Tedrus GMAS, Fonseca LC, Letro GH, Bossoni AS, Samara AB. Dmentia and mild cognitive impairment in patients with Parkinson's disease, Arq Neuropsiquiatr 2009;67:423-427.

2. Goetz CG, Emre M, Dubois B. Parkinson's disease dementia: definitions, guidelines, and research perspectives in diagnosis. Ann Neurol 2008;64(Suppl 2):S81-S92.

3. Caviness JN, Driver-Dunckley E, Connor DJ, et al. Defining mild cognitive impairment in Parkinson's disease. Mov Disord 2007;22:1215-1216.

4. Bosboom JL, Stoffers D, Wolters ECh. Cognitive dysfunction and dementia in Parkinson's disease. J Neural Transm 2004; 111:1303-1315.

5. Piovezan MR, Teive HAG, Piovesan EJ, Mader MJ, Werneck LC. Cognitive function assessment in idiopathic Parkinson's disease. Arq Neuropsiquiatr 2007;65:942-946.

Hélio A.G. Teive, MD, PhD

Mauro R. Piovesan, MD, MsC

Lineu César Werneck, MD, PhD

Serviço de Neurologia do HC da UFPR

\section{THE AUTHORS' REPLY}

We are grateful to Teive et al. for their interest and comments with respect to our article.

Cognitive dysfunctions are common in PD and comprehend attention, executive, visuospatial and memory deficits, but in the presence of dementia, behavioral symptoms such as affective changes, hallucinations, and apathy are frequent'.

With respect to the mechanisms involved in the genesis of cognitive dysfunctions in PD, in addition to those resulting from frontal dysfunction, secondary to the cortical and subcortical changes due to dopaminergic and cholinergic deficiencies, other mechanisms resulting from Lewy body-type degeneration in various cortical and limbic structures could be present $t^{1,2}$. Thus the type of memory deficit found in our study, with difficulty in both the recall and recognition of word lists, suggests the existence of mechanisms analogous to the memory deficit in Alzheimer's disease.

With respect to studies carried out in Brazil concerning the cognitive aspects of PD, various aspects have been considered. Amongst these, Piovesan et al. ${ }^{3}$ found statistically significant differences between PD patients and controls in terms of cognitive function (MMSE), verbal, executive and visuospatial functions and attention deficits. They also evaluated the occurrence of depression and showed correlation with some cognition aspects.

In our study we also found differences from the control group for all the variables analyzed, which included memory tests with word list recall and recognition. We are unaware of any other national study that evaluated the occurrence of mild cognitive impairment and dementia in PD patients.

Other studies evaluated specific cognition aspects, such as executive ${ }^{4}$ and visuospatial ${ }^{5}$ tests, in a more profound way, and pointed out there was heterogeneity of the results within the respective categories, which could correspond to the multiplicity of functions involved in these, as also they indicated the lack of instrument validation in our medium.

\section{REFERENCES}

1. Emre M, Aarsland D, Brown R, et al. Clinical diagnostic criteria for dementia associated with Parkinson's disease. Mod Disord 2007;12:1689-1707.

2. Farlow MR, Cummings J. A modern hypothesis: the distinct pathologies of dementia associated with Parkinson's disease versus Alzheimer' disease. Dement Geriatr Cogn Disord 2008; 25:301-308.

3. Piovezan MR, Teive HAG, Piovesan EJ, Mader MJ, Werneck LC. Cognitive function assessment in idiopathic Parkinson's disease. Arq Neuropsiquiatr 2007;65:942-946.

4. Sobreira EST, Pena MCS, Silva Filho JH, et al. Executive cognitive tests for the evaluation of patients with Parkinson's disease. Dement Neuropsychol 2008;2:206-210.

5. Pena MCS, Sobreira EST, Souza CP, Oliveira GN, Tumas V, Vale FAC. Vsuospatial cognitive tests for the evaluation of patients with Parkinson's disease. Dement Neuropsychol 2008;2:201-205.

Gloria Maria Almeida Souza Tedrus,

Lineu Corrêa Fonseca

Grace Helena Letro

Alexandre Souza Bossoni

Adriana Bastos Samara 